\title{
1 Innate triggering and antiviral effector functions of activin A
}

2

3 Al-Hourani K. ${ }^{*}$, Ramamurthy $\mathrm{N}^{*}$, Marchi E*, Eichinger RM, Lee LN, Fabris P, Klenerman P, 4 Drakesmith $\mathrm{H}$.

5

6 *These authors contributed equally to the study 


\section{Abstract}

8 First-line defence against viral infection is contingent upon rapid detection of conserved viral

9 structural and genomic motifs by germline-encoded pattern recognition receptors, followed

10 by activation of the type I IFN system and establishment of an intracellular antiviral state.

11 Novel antiviral functions of bone morphogenetic protein and related activin cytokines, acting

12 in conjunction with, and independently of, type I IFN, have recently been described. Activin A

13 mediates multiple innate and adaptive immune functions - including antiviral effects.

14 However, how such effects are mediated and how activin might be triggered by viral infection

15 have not been defined. Here we addressed this in vivo and in vitro, in humans and mice.

17 Transcriptomic analyses delineated strikingly congruent patterns of gene regulation in 18 hepatocytes stimulated with recombinant activin $A$ and IFNa in vitro. Activin A mRNA, 19 encoded by INHBA, is induced upon activation of RIG-I, MDA5 and TLR7/8 viral nucleic acid 20 sensors in vitro, across multiple cell lines and in human peripheral blood mononuclear cells.

21 In vivo, infection of mice with influenza A also upregulated Inhba mRNA in the lung; this local 22 upregulation of Inhba is retained in MAVS knockout mice, indicating a role for non-RIG-I-like 23 receptors in its induction. Activin induction and signalling were also detectable in patients 24 with chronic viral hepatitis.

26 Together, these data suggest Activin A is triggered in parallel with type I IFN responses and 27 can trigger related antiviral effector functions. This model has implications for the 28 development of targeted antiviral therapies, in addition to revealing novel facets of activin 29 biology. 


\section{Introduction}

34 First identified as a regulator of ovarian folliculogenesis, activin $A(1)$, a heterodimeric

35 assembly of inhibin $\beta_{A}$ subunits (encoded by the INHBA gene), is implicated in diverse

36 biological processes. In terms of innate immune functionality, activin $A$ is induced in

37 peripheral blood mononuclear cells exposed to pro-inflammatory stimuli including TNFa,

38 GM-CSF and IFNy(2); neutrophil granules are also rich sources of pre-formed activin A

39 protein(3). In vivo, the induction kinetics of activin A in response to systemic inflammatory

40 stimuli have been examined following intravenous LPS exposure: serum activin A protein

41 increases rapidly during endotoxaemic shock in mice, with administration of its soluble

42 antagonist follistatin (FST) sufficient to reduce subsequent mortality(4). Clinically, in H1N1

43 influenza-infected patients admitted to intensive care, elevated serum levels of both activin A

44 and FST were detected, and correlated with degree of respiratory distress(5).

46 Recent observations have shown an antiviral function of the TGF $\beta$-superfamily cytokines the 47 bone morphogenetic proteins (BMP) and the activins, both transduced via SMAD 48 transcription factors, a phenomenon elucidated via examination of the mutually antagonistic 49 interactions between Hepatitis C Virus (HCV) and the BMP/SMAD signalling axis. Activin A 50 exerts dose-dependent antiviral effects against an in vitro HCV genomic replicon(6). 51 Additionally, in vitro antiviral functions of activin A against Zika virus, a flavivirus akin to HCV, 52 and also against Hepatitis B Virus (HBV), a structurally distinct hepadnavirus(6) have been 53 reported.

55 While activin proteins can mediate antiviral effects against multiple viruses in vitro, 56 upregulation of activins in response to viral infection, akin to the rapid induction of type I IFN, 57 has not previously been described. Three classes of pattern recognition receptor (PRR) 58 sense viral nucleic acids: RIG-I-like receptors (RLR); Toll-like receptors (TLR); and the 59 cGAS-STING axis for detection of cytosolic DNA. RIG-I, the prototypic RLR detects 5'- 
60 triphosphate and diphosphate moieties associated with non-self $R N A(7,8)$. The RLR family

61 also includes MDA5, a RIG-I paralogue demonstrating length-dependent activation by

62 dsRNA(9).RLR activation drives the oligomerization of the adaptor protein MAVS, the

63 essential factor for downstream type I IFN induction(10).

64

65 In humans, four endosomal TLRs are sensitive to non-self nucleic acids:TLR3, TLR7, TLR8

66 and TLR9(11). TLRs comprise an ectodomain conferring ligand specificity;a transmembrane

67 region; and a cytosolic Toll/IL-1 receptor that ultimately activates IRAK, IKK and TBK1

68 kinases (11).

69

70 In this study, we first used microarray analysis and RNA sequencing to analyse the effects of

71 activin $\mathrm{A}$ upon hepatocytes at the transcriptional level and examine its intersection with the

72 type I IFN axis. We next addressed whether activin A transcription is induced upon both

73 PRR activation and viral infection, both in vitro and in vivo, in addition to in part delineating

74 the mechanistic basis for this phenomenon. Overall these data indicate that Activin plays a

75 role in virus infections as part of the innate response.

76

77 Results

78

81 As shown in Fig 1 A, in order to better understand the effect of Activin A on hepatocytes, we

82 used microarray analysis of the RNA isolated from HepaRG cells activated with 10nM Activin

83 A for $24 \mathrm{hrs}$. Differential analysis of the expression data show that 184 genes were

84 upregulated and 168 number of genes were down-regulated with a minimum of 1 log fold

85 change. Table 1 shows the top 40 list of differentially regulated genes flimma, paired t test

$86 \mathrm{p}<0.001 \mathrm{lfc}>1$ ). Metacore analysis (Clarivate Analytics) of the differentially regulated genes

87 show that genes in Type 1 alpha/beta signalling pathway were predominantly upregulated, 
88 followed by MHC Class I presentation and Response to RNA viral infection (Fig 1 B).

89 However, Activin A seems to be downregulating genes in the cell cycle pathway (Fig 1C).

\section{Activin A stimulates ISGs in hepatocyte cell lines}

93 Intrigued by the ability of Activin A to up-regulate ISG in the absence of Interferons, we

94 looked to address whether Activin A increases ISG levels by regulating IFN expression in the

95 HepaRG hepatocyte cell line over a $72 \mathrm{hr}$ period (Fig. 1D). qPCR analysis validated the

96 increase in ISG expression observed in microarray and RNA sequencing. HepaRG cells

97 stimulated with Activin A showed an increase in expression of various ISGs relative to un-

98 stimulated cells (Fig 1D). However, when tested on other cell lines, namely Huh7

99 hepatoma-derived cells and the immortalised transformed hepatocyte cell line HHL12 (12),

100 we observed that the pattern on gene expression over time is not consistent and individual

101 cell lines behave differently to Activin A in terms of ISG expression (Fig. 1E) The primer

102 pairs used for the qPCR are detailed in the supplementary information (Supplementary

\section{Table 1).}

105 RNA-sequencing confirms results of microarray data analysis on hepatocytes

\section{6 stimulated with Activin A}

108 We corroborated our data obtained by microarray analysis by performing a new RNA

109 sequencing experiment on a separate experiment with hepatocytes stimulated with Activin A.

110 We then performed a Gene set enrichment analysis (GSEA) comparing both experiments.

111 GSEA shows high correlation in gene expression by both methods of analysis. The entire set

112 of Activin vs Untreated up-regulated genes detected in the RNA-Seq experiment $(n=2571$,

113 data processed with DESeq2 package(13)) were found highly enriched compared to the

114 microarray data (Fig 2a).

115 
116 To show that ISG were upregulated in response to activin induction, we performed a GSEA

117 using a large set of Interferon signatures publicly available in MsigDB database(14). We

118 selected 110 gene sets querying all curated databases (e.g Reactome, GO, etc.) and

119 reported gene expression data from immunologic and cancer studies (e.g. Up-regulated

120 genes on cells stimulated with IFNa) in MsigDB. We found that 88 of all 110 gene sets were

121 up-regulated in Activin treated samples, of which 24 gene sets were significantly enriched at

122 FDR $<25 \%, 10$ gene sets were at nominal $p$-value $<0.01$, and 19 gene sets were significant

123 at nominal $p$-value $<0.05$. The remaining $22 / 110$ gene sets were enriched in Untreated

124 samples without any statistical significance. Fig $\mathbf{2 b}$ and Fig $\mathbf{2 c}$ shows two examples of

125 public IFN signatures enriched in up-regulated genes in our Activin vs Untreated samples (in

126 RNA-Seq data after voom transformation in limma R package(15)).

128 Activity of Activin A is independent of IFNa

130 We have previously shown that recombinant Activin A protein exerts a dose dependent

131 antiviral effect(6). We first explored the transcriptional pathways and show that Activin $A$ is

132 able to induce ISG signalling in the presence of B18R, a type I IFN binding protein derived

133 from Western Reserve Strain Vaccinia virus, in Huh7 cells (Fig. 3A). However, B18R is able

134 to inhibit RNA expression of ISG in response to IFNa (Fig. 3B). In addition, supernatants of

135 hepatocyte (Huh7) cells stimulated with Activin A did not show the presence of IFNA2 (Fig

$1363 \mathrm{C})$ supporting the view that Activin A acts independently of type I IFN. Additionally it was

137 observed that the antiviral functions of Activin A does not require signalling via the type I IFN

138 receptor (IFNAR), being unaffected by ruxolitinib (16) a pharmacological inhibitor of the

139 JAK1 kinase downstream of IFNAR [supplementary figure 1 A-C].

140

141 Evidence of in vivo expression of Activin A in viral infection 
142 To further examine whether Activin A is expressed during the course of viral infection in vivo,

143 we performed PCR to detect Activin A mRNA expression levels in livers and ELISA to detect

144 Activin a protein in serum of patients with HCV infection. Fig 4A. shows that Activin A levels

145 in inflamed liver samples from HCV patients were significantly increased. Significant

146 increases in Activin A protein levels in the non-responders to IFN therapy were observed

147 compared to controls (Fig. 4B). To assess whether Activin A could also be induced in an

148 acute viral infection we inoculated mice with MCMV intravenously. In this model Activin A

149 was detected at 3 days post-infection (Fig 4C). These data indicate that acute and chronic

150 viral infections both lead to induction of Activin A in vivo.

\section{Activin A is induced by PRR activation in human peripheral blood mononuclear cells}

152 In order to explore the signalling mechanisms responsible for activin A induction by

153 infectious stimuli, freshly-isolated human peripheral blood mononuclear cells, which express

154 a broad repertoire of cytosolic and endosomal viral sensors, were transfected transfection

155 with IVT-RNA or poly(I:C). These stimuli, detected by RIG-I and MDA5 respectively(17),

156 elicited a statistically significant upregulation of INHBA mRNA (Fig. 5A), mirrored by

157 significant transcriptional induction of $M X 1$ and IFI6, a pleiotropic antiviral effector (Fig. 5B,

158 5C).

159

160 Incubation with the synthetic guanidine base analogues R837 and R848 (TLR7 and TLR7/8

161 stimuli respectively) at $1 \mu \mathrm{g} / \mathrm{mL}$ resulted in approximately 15 -fold and 50 -fold increases in

162 INHBA expression respectively (Fig. 5D), implying a role for TLR7 in INHBA induction. This

163 was parallel by robust induction of $M X 1$, encoding the broadly antiviral GTPase MX1 (Fig.

164 5E). Statistically significant INHBA upregulation following incubation with ssRNA40 at

$1651 \mu \mathrm{g} / \mathrm{mL}$ and $5 \mu \mathrm{g} / \mathrm{mL}$ (figure 5F), with attendant induction of IFI6 (Fig. 5G), suggesting a

166 role for TLR8 in INHBA induction in vitro.

167 
168 Recapitulating these observations with live viral inocula, incubation of human CD14+

169 monocytes with HBV and HCV (multiplicity of infection $0.5-1.0$ ) for 16 hours resulted in

170 significant induction of INHBA mRNA and detectable activin A protein in the culture

171 supernatant (Fig. $5 \mathbf{H})$.

172

173 INHBA mRNA is upregulated by ssRNA virus infection in vitro

175 Having clarified the similarity of activin A and type I IFN in terms of patterns of antiviral gene

176 regulation in clinical infection models and also looked at its induction by synthetic immune

177 stimuli, our attention turned to the possibility that activin itself is induced by viral infection. In

178 RIG-I/TLR3 replete lung adenocarcinoma-derived A549.gfp cells, infection with the ssRNA

179 paramyxovirus Sendai virus $(\mathrm{SeV})$ results in a statistically significant, dose-dependent

180 induction of INHBA mRNA [figure 6A], paralleled by a strong induction of $M X 1$ [figure 6B].

181 SeV encodes a 546nt immunogenic motif within its 5'-UTR and intact RIG-I, but not MDA5,

182 signalling is necessary for IRF3 phosphorylation and subsequent ISG induction in vitro(18).

183

184 To further clarify the PRR requirements for INHBA induction by SeV, Huh7 and Huh7.5

185 hepatoma-derived cells were infected with a titration of SeV, and expression of INHBA, MX1

186 and genomic SeV quantified by RT-qPCR. The RIG-I isoform expressed by Huh7.5 encodes

187 an N-terminal point mutation that uncouples viral sensing from downstream signalling (19).

188 Huh7 cells and their derivatives are therefore deficient for TLR3 expression.

189 In RIG-I replete Huh7 cells, SeV infection elicits titre-dependent INHBA upregulation [figure

$1906 \mathrm{C}$ ], paralleled by strong induction of the IFN-dependent target gene MX1 at 24 hours post

191 infection [figure 6D]. In Huh7.5.1 cells, however, neither INHBA nor MX1 message levels

192 were altered by SeV infection. Baseline expression of both INHBA and $M X 1$ is approximately

193 10-fold higher in uninfected Huh7.5.1 cells versus Huh7. SeV genomic RNA levels were 
194 comparable in both cell lines, although the signal in the uninfected conditions implies low-

195 level off-target amplification [figure 6E].

196 Moving on to consider in vitro flavivirus infection, type I IFN induction secondary to dengue 197 virus (DENV) is reportedly contingent upon MAVS-dependent RLR signalling(20). MDA5 is

198 likely to represent the principal sensor of DENV in Huh7.5.1 cells, being deficient in 199 functional RIG-I and TLR3(18,19). At 48 hours post infection, DENV infection in Huh7.5.1 200 cells results in statistically significant upregulation of mRNA encoding INHBA [figure 6F], 201 accompanied by a significant upregulation of $M X 1$ [figure $6 \mathrm{G}$ ].

202 We quantified INHBA expression in Huh7 cells incubated with recombinant IFNa-2a, and 203 established that INHBA mRNA is not induced by recombinant type I IFN in this cell line 204 [supplementary figure 1A]. Akin to the antiviral enhancement of type I IFN by BMP6, co205 incubation of OR6 HCV genomic replicon cells with recombinant activin A synergistically 206 enhances the antiviral effect of IFNa-2a, hinting at possible functional ramifications of viral 207 INHBA induction [supplementary figure $1 \mathrm{~B}-\mathrm{D}]$.

\section{Influenza A infection induces Inhba expression in the lungs of C57BL/6 mice}

209 Moving to in vivo infection models, we observed that Inhba expression is significantly 210 upregulated in the lungs of C57BL/6 mice infected with the influenza A [FLUAV] strain $211 \mathrm{PR} / 8 / 34$ for 72 hours compared to uninfected controls [figure 7A]. This induction correlates 212 tightly with that of Isg15, encoding a pleiotropic, IFN-stimulated antiviral effector(21) $\left(\mathrm{R}^{2}=\right.$ $2130.9271, \mathrm{p}=0.0020$ ) [figure 7B-7C]. In the liver, however, Inhba expression is significantly 214 suppressed by FLUAV [figure 7D], despite no alteration in Isg15 expression [figure 7E]. 215 Suppression of hepatic Inhba expression in the presence of a systemic inflammatory 216 response has been previously reported, albeit in the context of LPS administration $(22,23)$, 217 not viral infection. 
218 Upon intranasal infection with titrating doses of FLUAV, Inhba is dose-dependently

219 upregulated at 48 hours post infection, reaching maximal a three-fold increase with the

$2205 \times 10^{4}$ plaque forming unit (pfu) inoculum [figure 7F].

221 To gain a mechanistic insight into the PRR subsets responsible for Inhba induction during 222 intranasal FLUAV infection, we compared Inhba expression in lung lysates from both 223 wildtype (WT) and Mavs knockout (KO) mice. These animals cannot transduce RLR 224 signalling (24). In wildtype and Mavs KO mice, Inhba expression was significantly increased 225 by intranasal administration of $5 \times 10^{4}$ pfu FLUAV at 48 hours post-infection,albeit by a 226 smaller fold change in the transgenic animals [figure 7G]. Similarly, the magnitude of both 227 Inhba and Isg15 induction was lower in Mavs KO compared to WT mice, although this 228 difference did not attain statistical significance [figure $7 \mathrm{H}$ ]. These data suggest a function of 229 Mavs-independent pattern recognition in Inhba induction by FLUAV.

\section{Discussion}

232 An antiviral function of the BMP/activin-SMAD signalling axis has been described in the 233 context of viral infection(6). Here, we show that gene expression signatures associated with 234 activin stimulation are induced in parallel to those downstream of type I IFN in a clinically 235 important viral disease, namely chronic HCV infection. Additionally, we provide evidence for 236 induction of activin A, encoded by the INHBA gene, in multiple models of viral infection, both 237 in vitro and in vivo.

239 The transcriptomic analyses described here suggest that stimulation of antiviral effector 240 genes classically regarded as "interferon-stimulated" may not be an exclusive feature of IFN, 241 as both BMP6 (6) and activin A are able to upregulate ISG in the absence of exogenous 242 IFNa and ISG induction occurs as a summation of diverse unrelated signalling events. 243 Together with the observed antiviral properties of recombinant activin A, we propose that its 
244 transcriptional induction downstream of viral sensing may represent a hitherto undescribed 245 antiviral feedback response, initiated upon detection of infection and acting to curtail viral 246 replication.

$247 \mathrm{Wu}$ and colleagues report that in the resting state, murine alveolar and bronchial 248 macrophages stain positively for activin A protein; during LPS endotoxaemia, activin A 249 positive neutrophils aggregate in the perialveolar spaces (23). This reported accumulation of 250 activin A-rich neutrophils in the lung correlated with reduced numbers in the bone marrow, 251 consistent with migration into the systemic circulation. It was posited that this activin $A$ 252 induction occurred at the post-transcriptional level, being ablated by cyclohexamide but 253 unaffected by the transcription initiation inhibitor actinomycin D (23). In contrast, our data 254 obtained with FLUAV demonstrate transcriptional upregulation of Inhba, with respiratory 255 epithelia and alveolar macrophages being equally plausible candidate sources.

256 In vivo, Inhba mRNA is upregulated in the lungs of mice following intranasal FLUAV 257 infection. This induction is not abrogated upon Mavs knockout, implying a contribution of 258 Mavs-independent viral sensing. Data from human PBMC in vitro indicate that TLR7/8 259 activation - that is, independent of MAVS - is sufficient for INHBA upregulation. As such, 260 definitive characterization of Inhba induction in vivo will require examination of mice deficient 261 in Tbk1, the point of RLR and TLR convergence upon IRF3.

262 Our observations of INHBA induction in experimental infection systems correspond with 263 clinical reports of elevated serum activin A protein in both acute influenza A infection, where 264 it correlates with the degree of respiratory distress, and in chronic HCV $(5,25)$. Interpretation 265 of these patient studies is complicated by the well-established roles of activin A and TGF $\beta$ 266 signalling in tissue fibrosis (26); it is impossible to dissect whether elevated activin A occurs 267 immediately downstream of viral sensing or as a mediator of fibrotic immune pathology. 
268 Given the described antiviral properties of exogenous activin proteins and manipulation of

269 the activin/SMAD signalling axis(6), these data nevertheless provide an essential first step in

270 the clarification of activin A's endogenous role in the innate response to viral infection.

\section{Acknowledgements:}

273 The authors would like to thank Dr. Azim Ansari for useful discussions; Dr Jo Miller ,

274 Professor Nicole Zitzmann, Dr Rachel Rigby and Professor Jan Rehwinkel for technical

275 advice and experimental assistance. The authors also thank the High-Throughput

276 Genomics Group at the Wellcome Trust Centre for Human Genetics (funded by Wellcome

277 Trust grant reference 090532/Z/09/Z and MRC Hub grant G0900747 91070) for the

278 generation of the Sequencing and micro-array data). This research was supported by the

279 Wellcome Trust (WT109965MA) NIHR Senior Fellowship (PK), NIH U19 1082360. The

280 authors wish to acknowledge the BRC Oxford GI Biobank in collecting and making available

281 the samples/data used in the generation of this publication. The research was supported by

282 the National Institute for Health Research (NIHR) Oxford Biomedical Research Centre

283 (BRC). The views expressed are those of the authors and not necessarily those of the NHS,

284 the NIHR or the Department of Health.

285 The data that support the findings of this study are available from the corresponding author

286 upon reasonable request.

\section{Author contributions:}

$289 \mathrm{KAH}, \mathrm{NR}, \mathrm{PK}$ and $\mathrm{HD}$ designed the experiments. KAH, NR and RME performed the

290 experiments and wrote the manuscript, LNL performed in vivo MCMV infections, PF

291 provided liver biopsy tissue and EM performed the bioinformatic analysis. 


\section{Materials and Methods:}

\section{Transcriptomics pipeline: extraction and sample preparation}

298 Total RNA was prepared after stimulation or that of untreated controls with the RNeasy Mini/

299 Plus Micro Kit (Qiagen, UK cat\# 74106/ 74034). Stimulated cells were lysed in buffer RLT

300 (Qiagen) and homogenized with a QIAshredder (Qiagen). RNA was quantified spectro301 photometrically and the quality of RNA preparations for Illumina microarray analysis was 302 checked with an Agilent Technologies 2100 Bio-analyzer.

304 Two-step reverse transcription was performed on the RNA using AppScript cDNA synthesis 305 kit (Appleton Woods) and quantitative PCR performed using the Roche Light Cycler 480.

306 Approximately 500ng of total RNA from cells was reverse transcribed using the AppScript 307 cDNA synthesis kit. Briefly, total RNA was mixed with kit components App RTase and App 308 cDNA mix to a final volume of 20 microlitrel. The mix was then incubated at $42^{\circ} \mathrm{C}$ for 30 mins.

309 At the end of the reaction RTase was inactivated by heating the reaction to $85^{\circ} \mathrm{C}$ for 10 310 mins.

312 For RT-qPCR validation, 5 ul of a 1/20th dilution of resultant cDNA was used as a template 313 for qPCR using the Roche light cycler 480 to detect expression of selected ISGs. Primers 314 were designed using the Roche Universal Probe Library system. Relative gene expression 315 was calculated using the comparative cycle threshold method (27) normalized to expression 316 of the house-keeping gene GAPDH and expressed relative to a mock treated sample.

\section{Transcriptomics pipeline: microarray analysis}


319 500ng of total RNA was used for microarray analysis and was performed by the Oxford

320 Genomic Centre at the Wellcome Trust Centre for Human Genetics, University of Oxford.

321 The quality of the RNA was to have a RNA integrity number (RIN) greater than 7 and $32228 \mathrm{~S} / 18 \mathrm{~S}$ ratio of greater than 1.6.

324 A whole genome gene expression analysis was performed utilizing the Human HT12v4.0 325 Expression Beadchip. The RNA was converted to biotin labelled cRNA which was then 326 hybridised to the chip. The hybridised chip was then scanned using llumina's iScan scanner.

327 The gene expression profile was then created using illumina's GenomeStudio software.

\section{Transcriptomics pipeline: RNA sequencing analysis}

331 The mRNA fraction was selected from the total RNA provided before conversion to cDNA. 332 dUTP was incorporated during second Strand cDNA synthesis. The cDNA was end333 repaired, A-tailed and adapter-ligated. Before amplification the samples were uridine 334 digested. The prepared libraries were then size selected, multiplexed and QC'ed before 335 paired end sequencing over one rapid run. The data was then aligned to the reference and 336 quality checked.

\section{Cell lines}

340 OR6 cells (kindly from Prof Raymond Chung [MGH, MA, USA] with permission of Prof Kato 341 and Dr Ikeda [Okayama University, JP]) were cultured in Dulbecco's Modified Eagle's 342 Medium (DMEM), supplemented with 10\% Foetal Calf Serum (FCS) (PAA, , AT or Lonza, $343 \mathrm{CH}$ ), $100 \mathrm{U} / \mathrm{mL}$ penicillin (Sigma-Alrdich, USA), and $0.1 \mathrm{mg} / \mathrm{mL}$ streptomycin (Sigma-Aldrich) 344 and 2mM L-glutamine (Sigma-Aldrich): hereafter referred to as "D10". OR6 cells are stably 345 transfected with a full-length HCV genomic replicon in tandem with a Renilla luciferase 
346 reporter and neomycin resistance cassette. OR6 cells were under negative selection with

347 G418 $100 \mu \mathrm{g} / \mathrm{mL}$ (Sigma-Aldrich).

349 Huh7 (ATCC, USA) and Huh7.5.1 (kindly from Prof Raymond Chung [MGH, MA, USA]) cells 350 were maintained in D10.

352 HepaRG were cultured at $37^{\circ} \mathrm{C}, 5 \% \mathrm{CO}_{2}$ in Williams' E medium (with Glutamine) (Gibco353 Invitrogen) supplemented with glutamine (2 $\mathrm{mM})$, penicillin/streptomycin $(50 \mathrm{U} / \mathrm{mL})$, 354 gentamycin $(20 \mu \mathrm{g} / \mathrm{mL})$, insulin bovine $(5 \mu \mathrm{g} / \mathrm{mL}$, Roche-Boehringer-Manheim, France), 355 hydrocortisone hemisuccinate $\left(7 \times 10^{-5} \mathrm{M}\right.$, Roche-Boehringer-Manheim) and FCS (10\% 356 selected, non-decomplemented, Fetaclone II-Hyclone-PERBIO France). The cells were 357 allowed to differentiate in the presence of $2 \%(\mathrm{v} / \mathrm{v})$ DMSO to the medium.

359 A549.gfp cells (a kind gift from Prof Richard Randall, University of St Andrews, UK) were 360 maintained in in D10.

\section{Patients Samples}

363 HCV patient samples were obtained from patients enrolled at the JR Hospital, Oxford and 364 samples stored under protocol $16 / \mathrm{YH} / 0247$. Liver biopsy samples were collected prior to 365 commencement of anti-viral therapy and were graded and staged using ISHAK scoring at S.

366 Bortolo Hospital Vicenza, Italy. RNA extraction, reverse transcription and qRT-PCR was 367 performed as described below. Ethical approval for use of the biopsy samples was obtained 368 from the relevant local ethics committees. Informed written consent was obtained from all 369 patients involved.

\section{Peripheral blood mononuclear cells extraction}


374 Whole blood was procured from nine healthy volunteer donors in accordance with local

375 ethical policy and practices, as described previously(28,29). Blood samples were heparin-

376 treated before immediate layering onto a Ficoll-Pacque Plus ${ }^{\mathrm{TM}}$ (GE Healthcare Life

377 Sciences, USA) density centrifugation gradient in a 1:1 volume ratio. Samples were

378 centrifugedand the buffy coat layer aspirated with a pipette. Buffy coat cells were washed

379 three times in $2.5 \mathrm{mM}$ EDTA-PBS at $4^{\circ} \mathrm{C}$ before dilution and maintenance in RPMI-1640

380 supplemented with 10\% FCS (PAA, AT or Lonza, CH) $2 \mathrm{mM}$ glutamine, $100 \mathrm{U} / \mathrm{mL}$ penicillin,

$3810.1 \mathrm{mg} / \mathrm{mL}$ streptomycin (all Sigma-Aldrich).

\section{Enrichment of CD14 monocytes}

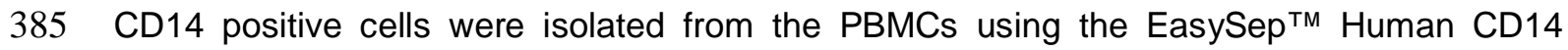
386 Positive Selection Kit by STEMCELL Technologies ${ }^{\text {TM }}$ (Cat\# 18058).

\section{Synthetic nucleic acids and base analogues}

390 High molecular weight poly(I:C) [cat I.D. t|r|-pic], poly(dA:dT) [cat. I.D: t|rl-patn], E. coli K12 391 dsDNA [cat. I.D. t|rl-ecdna], ODN 2216 [cat. I.D. t|rl-2216], R837 [cat. I.D. t|rl-imqs], R848 392 [cat. I.D. tIrl-r848] and ssRNA40/LyoVec ${ }^{\text {TM }}$ [cat. I.D. tlrl-Irna40] were purchased from 393 InvivoGen (FR). IVT-RNA transcribed by the T7 RNA polymerase and corresponding to 394 nucleotides 1-99 of the neomycin phosphotransferase gene was synthesized as described in 395 (30) (kindly from Prof Jan Rehwinkel [University of Oxford, UK]).

\section{Transfection and stimulation of unfractionated PBMC and CD14+ monocytes}

399 Human PBMC were extracted from healthy donors as described in section 2.1.6 and plated 400 at $1 \times 10^{6}$ cells/well of a $24-$ well plate. PBMC were transfected with IVT-RNA and poly(I:C) 401 using Lipofectamine® LTX with PLUS ${ }^{\text {TM }}$ (Life Technologies). R837, R848, ODN 2216 and 
402 ssRNA40 were added to the culture medium in the absence of transfection reagent to the 403 end-point concentrations specified.

$404 \mathrm{CD} 14+$ cells were plated at at $1 \times 10^{6}$ in $300 \mu \mathrm{R} 10$ medium per well and stimulated with HBV 405 inoculum (final $\mathrm{MOI}=0.5-1$ ), $\mathrm{HCV}$ inoculum (final $\mathrm{MOI}=0.5-1$ ). Cells were incubated at $40637^{\circ} \mathrm{C}, 5 \% \mathrm{CO}_{2}$ for 16 hours. Cells and supernatants were harvested and frozen at $-20^{\circ} \mathrm{C}$ for 407 further analysis.

408

409 In vitro Sendai virus (SeV) Infection

411 Cantell strain Sendai Virus [cat. I.D. ATCC® VR-907 ${ }^{\mathrm{TM}}$ ] was purchased from ATCC. A549, 412 Huh7 or Huh7.5 cells were plated at $5 \times 10^{4}$ cells/well in 12 -well plates and incubated at $37^{\circ} \mathrm{C}$ 413 for $24 \mathrm{~h}$. Immediately prior to infection, D10 was aspirated and the monolayers washed twice 414 times with PBS, before addition of $\mathrm{SeV}$ at $\mathrm{MOI}$ 0.5, MOI 1 and MOI 5 in 500uL serum-free 415 DMEM. Cells were incubated with $\mathrm{SeV}$ for $2 \mathrm{~h}$ at $37^{\circ} \mathrm{C}$, before removal of the virus, two PBS 416 wash/aspirations and replenishment with D10.

418 In vitro dengue virus (DENV) infection

420 Huh7.5 cells were plated at $3 \times 10^{5}$ cells/well in 12 -well plates and allowed to adhere. Cells 421 were incubated with DENV2 (strain 16681) in serum-free DMEM for $2 \mathrm{~h}$ at room temperature 422 with gentle agitation, followed by removal of virus, washing with PBS and incubation at $37^{\circ} \mathrm{C}$ 423 for $48 \mathrm{~h}$ in D10.

\section{In vivo influenza A virus (FLUAV) infection}

427 For figure 4A-E, 6 week old female C57BL/6 mice were anaesthetized and infected 428 intranasally (i.n.) with 3.5 haemagglutinating units FLUAV PR/8/34 (H1N1), kindly provided 429 by Prof John Skehel (NIMR, Mill Hill, UK). Control mice were administered PBS i.n. Mice 
430 were sacrificed at 72 h.p.i. by asphyxiation. Whole lungs were immediately lysed in RLT

431 buffer (Qiagen) with $10 \mu \mathrm{L} / \mathrm{mL} \quad \beta$-mercaptoethanol and mechanically disrupted with a

432 TissueRuptor (Qiagen), before RNA extraction, cDNA synthesis and RT-qPCR quantification

433 of gene expression as described in section 2.2. Liver explants $\left(\sim 2 \mathrm{~mm}^{3}\right)$ were preserved in

434 RNAlater (Qiagen), before mechanical lysis with a TissueRuptor and RNA extraction, cDNA

435 synthesis and RT-qPCR quantification of gene expression.

437 For figure 4F-G, 6-week old female wildtype C57BL/6 mice were infected i.n. with increasing 438 titres of wildtype FLUAV PR/8/34 from $4 \times 10^{2}$ to $5 \times 10^{4} \mathrm{pfu}$. Control mice were administered 439 DMEM i.n. Mice were sacrificed at 48 h.p.i. by asphyxiation, followed by RNA extraction, 440 cDNA synthesis and RT-qPCR quantification of gene expression. After sacrifice, whole lungs 441 were removed and snap-frozen in liquid $\mathrm{N}_{2} .1 \mathrm{~mL}$ Tri Reagent (Sigma-Aldrich) was added to 442 each lung, before lysis with glass beads [cat. I.D. G8772] (Sigma-Aldrich) in a FastPrep Cell 443 Disrupter. RNA was extracted from the lungs with lysates with phenol-chloroform followed by 444 isopropanol precipitation Sacchi (31). RNA isolates were further purified, and depleted for 445 genomic DNA, by secondary extraction using the RNeasy Plus kit (Qiagen). cDNA synthesis 446 and RT-qPCR analysis of gene expression were conducted as described previously.

448 For figure 4H-I, 6-week old female C57BL/6 mice, both wildtype and MAVS KO (32) were 449 infected i.n. with $5 \times 10^{4}$ pfu wildtype FLUAV PR/8/34. Mice were sacrificed at 48 h.p.i. by 450 asphyxiation. Control mice were administered DMEM i.n. Whole lungs were harvested and 451 RNA extracted as per the preceding instructions, followed by cDNA synthesis and RT-qPCR 452 quantification of gene expression. Animal experimentation was in line with requirements 453 stipulated in the ARRIVE guideline checklist.

\section{RNA isolation and quantification}


457 Unless otherwise specified, cell lysates were homogenized with a QIAshredder column

458 (Qiagen) and RNA extracted with the RNEasy Mini Kit (Qiagen). RNA concentration was 459 determined with a NanoDrop 2000 Spectrophotometer (Thermo Fisher Scientific, MA, USA) 460 at $260 \mathrm{~nm}$. The quality of RNA preparations for Illumina microarray analysis and RNA 461 sequencing analysis was checked with an Agilent Technologies 2100 Bio Analyser.

\section{cDNA synthesis and RT-qPCR analysis}

cDNA was reverse-transcribed from template RNA either using a two-step reverse

466 transcription using AppScript cDNA synthesis kit (Appleton Woods) or using the High 467 Capacity RNA-to-cDNA kit (Applied Biosystems, USA).

469 All RT-qPCR reactions were either performed using an Applied Biosystems 7500 Fast Real470 Time PCR System (Applied Biosystems, USA). For TaqMan ${ }^{\mathrm{TM}}$ quantification, gene 471 expression was assessed with inventoried TaqMan $^{\mathrm{TM}}$ Gene Expression Assays (Applied 472 Biosystems, MA, USA) diluted in $\operatorname{TaqMan}^{\mathrm{TM}}$ Gene Expression Master Mix (Applied 473 Biosystems, USA). TaqMan ${ }^{\mathrm{TM}}$ inventoried assays used: GAPDH (glyceraldehyde 3474 phosphate dehydrogenase) Hs99999905_m1; INHBA (inhibin beta A) Hs01081598_m1; 475 MX1 (myxovirus resistance 1, mouse, homolog of) Hs00895598_m1; IFI6 (interferon-alpha476 inducible-protein 6) Hs00242571_m1; IFNAR2 (Interferon alpha, beta and omega, receptor 477 2) Hs01022061_m1; JAK1 (Janus kinase 1) Hs01026983_m1; STAT2 (Signal transducer 478 and activator of transcription 2), Hs01013123_m1; Hprt (hypoxanthine-guanosine 479 phosphoribosyltransferase) Mm01545399_m1; Inhba (inhibin beta A) Mm00434339_m1; 480 Isg15 (ubiquitin-like modifier ISG15) Mm01705338_s1; Trim14 (tripartite motif-containing 481 protein 14) Mm01352552_m1 or quantitative realtime PCR analysis was performed using the 482 Roche Light Cycler 480 instrument using AppProbe reagents (Appleton Woods). Primers 483 were designed using the Roche Universal Probe library system. Relative gene expression 484 was calculated using the comparative cycle threshold method(27)normalised to expression 
485 of the housekeeping gene GAPDH and expressed relative to a mock treated sample. The

486 primer list used in fig $1 \mathrm{D}$ and E, 2 A and B, 4 A are indicated in Supplementary Table 2.

488 Plasma cytokine and Activin A quantification

490 Concentrations of interferon alpha 2 in cell culture supernatants was measured using custom 491 multiplex immunoassay kits (GeniePlex, Ireland).

492 Concentration of Activin $A$ in human and mouse serum samples were performed using 493 sandwich ELISA- Activin A immunoassay kit from R\&D system (Cat DAC008).

495 Bioinformatic and Statistical Analysis.

497 The Illumina bead chip output files were processed and analysed using the R statistical 498 software $(v$ 2.11)(33) and statistical testing was performed using the Linear Models for 499 Microarray Analysis (limma) package and DESeq2(13,34). Differential gene expression 500 between the experimental groups was assessed by generating relevant contrasts 501 corresponding to the possible cell type comparisons. Raw $p$-values were corrected for 502 multiple testing using the false discovery rate controlling procedure of Benjamini and 503 Hochberg (35); adjusted p-values $<0.01$ were considered significant. Gene set enrichment 504 analysis methods (GSEA) are as described(36,37). Pathway representation was performed 505 using the MetaCore pathway analysis software from Clarivate Analytics. Unless otherwise 506 specified, data transformation and analysis was performed with Microsoft Excel (Microsoft 507 Inc., USA). Statistical analysis and data presentation were performed with GraphPad Prism 508 (GraphPad Software Inc., USA).

\section{References:}


512

1. Vale W, Rivier J, Vaughan J, McClintock R, Corrigan A, Woo W, et al. Purification and characterization of an FSH releasing protein from porcine ovarian follicular fluid. Nature. 1986 Jun;321(6072):776-9.

2. Shao L, Frigon Jr. NL, Sehy DW, Yu AL, Lofgren J, Schwall R, et al. Regulation of production of activin $A$ in human marrow stromal cells and monocytes. Exp Hematol. 1992;20(10):1235-42.

3. Chen Y, Wu H, Winnall WR, Loveland KL, Makanji Y, Phillips DJ, et al. Tumour necrosis factor-alpha stimulates human neutrophils to release preformed activin A. Immunol Cell Biol. 2011;89(8):889-96. doi: 10.1038/icb.2011.12. Epub 2011 Mar 29.

4. Jones KL, Mansell A, Patella S, Scott BJ, Hedger MP, de Kretser DM, et al. Activin A is a critical component of the inflammatory response, and its binding protein, follistatin, reduces mortality in endotoxemia. Proc Natl Acad Sci U S A. 2007;104(41):16239-44. Epub 2007 Oct 2.

5. Linko R, Hedger MP, Pettila V, Ruokonen E, Ala-Kokko T, Ludlow H, et al. Serum activin $A$ and $B$, and follistatin in critically ill patients with influenza $A(H 1 N 1)$ infection. BMC Infect Dis. 2014;14(doi):10.1186/1471-2334-14-253.

6. A. Eddowes L, Al-Hourani K, Ramamurthy N, Frankish J, T Baddock H, Sandor C, et al. Antiviral activity of bone morphogenetic proteins and activins. Nature Microbiology. 2018.

7. Hornung V, Ellegast J, Kim S, Brzózka K, Jung A, Kato H, et al. 5'-Triphosphate RNA Is the Ligand for RIG-I. Science. 2006 Nov 10;314(5801):994-7.

8. Goubau D, Schlee M, Deddouche S, Pruijssers AJ, Zillinger T, Goldeck M, et al. Antiviral immunity via RIG-I-mediated recognition of RNA bearing 5[prime]diphosphates. Nature. 2014 Oct 16;514(7522):372-5.

9. Kato H, Takeuchi O, Mikamo-Satoh E, Hirai R, Kawai T, Matsushita K, et al. Lengthdependent recognition of double-stranded ribonucleic acids by retinoic acid-inducible gene-I and melanoma differentiation-associated gene 5. Journal of Experimental Medicine. 2008;205(7):1601-10.

10. Xu L-G, Wang Y-Y, Han K-J, Li L-Y, Zhai Z, Shu H-B. VISA is an adapter protein required for virus-triggered IFN- $\beta$ signaling. Molecular Cell. 2005;19(6):727-40.

11. Kawai T, Akira S. Toll-like receptors and their crosstalk with other innate receptors in infection and immunity. Immunity. 2011 May;34(5):637-50.

12. Clayton RF, Rinaldi A, Kandyba EE, Edward M, Willberg C, Klenerman P, et al. Liver cell lines for the study of hepatocyte functions and immunological response. Liver International. 2005 Apr;25(2):389-402.

13. Love MI, Huber W, Anders S. Moderated estimation of fold change and dispersion for RNA-seq data with DESeq2. Genome Biology. 2014;15(12):550.

14. Liberzon A, Birger C, Thorvaldsdottir H, Ghandi M, Mesirov JP, Tamayo P. The Molecular Signatures Database (MSigDB) hallmark gene set collection. Cell systems. 2015 Dec;1(6):417-25. 
552

15. Ritchie ME, Phipson B, Wu D, Hu Y, Law CW, Shi W, et al. limma powers differential expression analyses for RNA-sequencing and microarray studies. Nucleic Acids Research. 2015 Jan 20;43(7):e47-e47.

16. Quintás-Cardama A, Vaddi K, Liu P, Manshouri T, Li J, Scherle PA, et al. Preclinical characterization of the selective JAK1/2 inhibitor INCB018424: therapeutic implications for the treatment of myeloproliferative neoplasms. Blood. 2010 Feb 3;115(15):3109-17.

17. Yoneyama M, Onomoto K, Jogi M, Akaboshi T, Fujita T. Viral RNA detection by RIG-Ilike receptors. Current Opinion in Immunology. 2015 Feb;32:48-53.

18. Li K, Chen Z, Kato N, Gale M, Lemon SM. Distinct Poly(I-C) and Virus-activated Signaling Pathways Leading to Interferon- $\beta$ Production in Hepatocytes. Journal of Biological Chemistry . 2005 Apr 29;280(17):16739-47.

19. Sumpter R, Loo Y-M, Foy E, Li K, Yoneyama M, Fujita T, et al. Regulating Intracellular Antiviral Defense and Permissiveness to Hepatitis C Virus RNA Replication through a Cellular RNA Helicase, RIG-I. Journal of Virology . 2005 Mar 1;79(5):2689-99.

20. Loo Y-M, Fornek J, Crochet N, Bajwa G, Perwitasari O, Martinez-Sobrido L, et al. Distinct RIG-I and MDA5 signaling by RNA viruses in innate immunity. Journal of Virology. 2008;82(1):335-45.

21. Zhao C, Collins MN, Hsiang T-Y, Krug RM. Interferon-induced ISG15 pathway: an ongoing virus-host battle. Trends in microbiology. 2013 Apr;21(4):181-6.

22. Besson-Fournier C, Latour C, Kautz L, Bertrand J, Ganz T, Roth MP, et al. Induction of activin $B$ by inflammatory stimuli up-regulates expression of the iron-regulatory peptide hepcidin through Smad1/5/8 signaling. Blood. 2012;120(2):431-9. doi: 10.1182/blood2012-02-411470. Epub 201.

23. Wu H, Chen Y, Winnall WR, Phillips DJ, Hedger MP. Acute regulation of activin A and its binding protein, follistatin, in serum and tissues following lipopolysaccharide treatment of adult male mice. American Journal of Physiology - Regulatory, Integrative and Comparative Physiology. 2012 Sep 15;303(6):R665-75.

24. Sun Q, Sun L, Liu H-H, Chen X, Seth RB, Forman J, et al. The Specific and Essential Role of MAVS in Antiviral Innate Immune Responses. Immunity. 2006 May;24(5):63342.

25. Refaat B, Ashshi A, El-Shemi A, AlZanbagi A. Effects of chronic hepatitis $C$ genotype 1 and 4 on serum activins and follistatin in treatment naïve patients and their correlations with interleukin-6, tumour necrosis factor-a, viral load and liver damage. Clinical and Experimental Medicine. 2014;1-10.

26. de Kretser DM, O'Hehir RE, Hardy CL, Hedger MP. The roles of activin A and its binding protein, follistatin, in inflammation and tissue repair. Molecular and Cellular Endocrinology. 2012 Aug 15;359(1-2):101-6.

27. Livak KJ, Schmittgen TD. Analysis of relative gene expression data using real-time quantitative PCR and the 2(-Delta Delta C(T)) Method. Methods (San Diego, Calif). $2001 ; 25(4): 402-8$.

28. Armitage AE, Pinches R, Eddowes LA, Newbold Cl, Drakesmith H. Plasmodium falciparum infected erythrocytes induce hepcidin (HAMP) mRNA synthesis by 
peripheral blood mononuclear cells. British Journal of Haematology. 2009 Dec 1;147(5):769-71.

29. Armitage AE, Eddowes LA, Gileadi U, Cole S, Spottiswoode N, Selvakumar TA, et al. Hepcidin regulation by innate immune and infectious stimuli. Blood. 2011 Oct;118(15):4129-39.

30. Rehwinkel J, Tan CP, Goubau D, Schulz O, Pichlmair A, Bier K, et al. RIG-I Detects Viral Genomic RNA during Negative-Strand RNA Virus Infection. Cell. 2010 Feb 5;140(3):397-408.

31. Chomczynski P, Sacchi N. Single-step method of RNA isolation by acid guanidinium thiocyanate-phenol-chloroform extraction. Analytical biochemistry. 1987 Apr;162(1):156-9.

32. Michallet M-C, Meylan E, Ermolaeva MA, Vazquez J, Rebsamen M, Curran J, et al. TRADD protein is an essential component of the RIG-like helicase antiviral pathway. Immunity. 2008 May;28(5):651-61.

33. Team RCD. R: A language and environment for statistical computing. Vienna, Austria: R Foundation for Statistical Computing; 2008.

34. Smyth GK. limma: Linear Models for Microarray Data BT - Bioinformatics and Computational Biology Solutions Using R and Bioconductor. In: Gentleman R, Carey VJ, Huber W, Irizarry RA, Dudoit S, editors. New York, NY: Springer New York; 2005. p. 397-420.

35. Benjamini Y, Hochberg Y. Controlling the False Discovery Rate $\square$ : a Practical and Powerful Approach to Multiple Testing When researchers tend to select pursuing multiple the ( statistically) and support of conclusions. An unguarded use in a greatly results of single-inference inc. JR Statist SocB. 1995;57(1):289-300.

36. Subramanian A, Subramanian A, Tamayo P, Tamayo P, Mootha VK, Mootha VK, et al. Gene set enrichment analysis: a knowledge-based approach for interpreting genomewide expression profiles. Proceedings of the National Academy of Sciences of the United States of America. 2005;102(43):15545-50.

37. Mootha VK, Lindgren CM, Eriksson K-F, Subramanian A, Sihag S, Lehar J, et al. PGC1alpha-responsive genes involved in oxidative phosphorylation are coordinately downregulated in human diabetes. Nature genetics. 2003;34(3):267-73. 


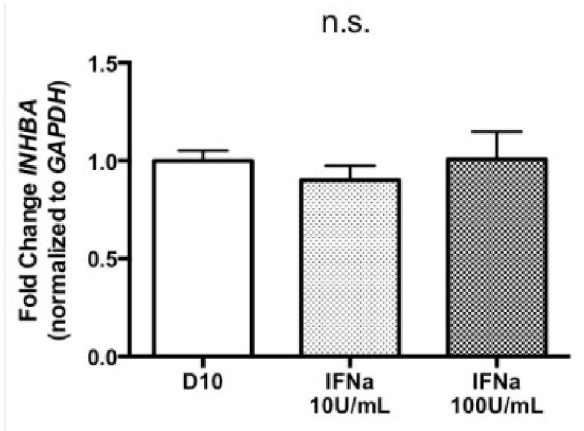

IFNa Dose

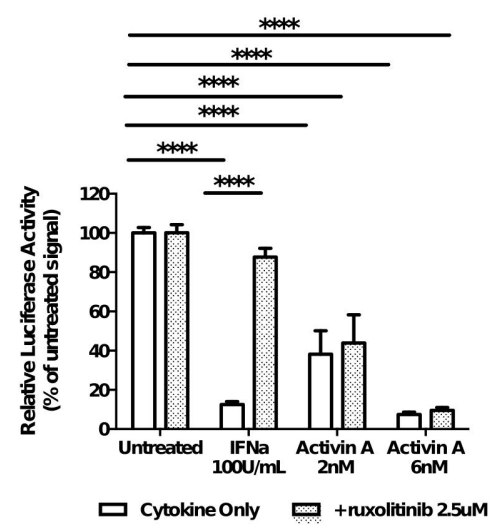

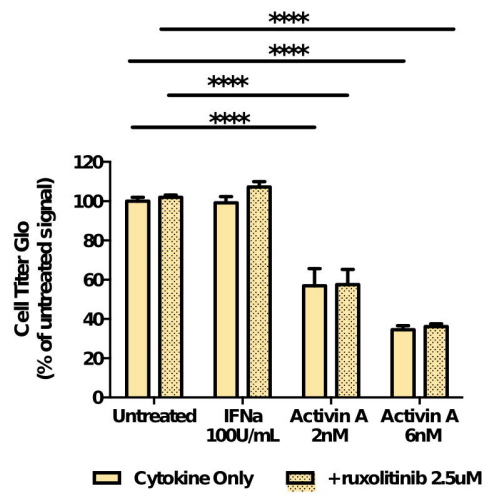

D
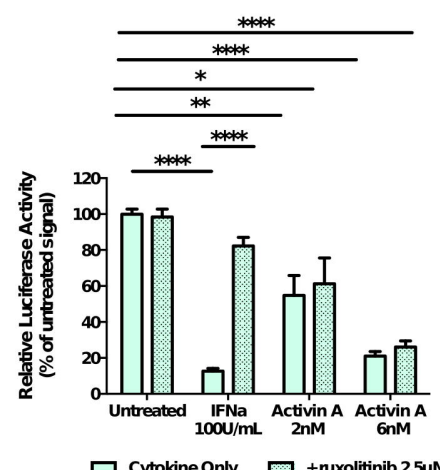

口 Cytokine Only 


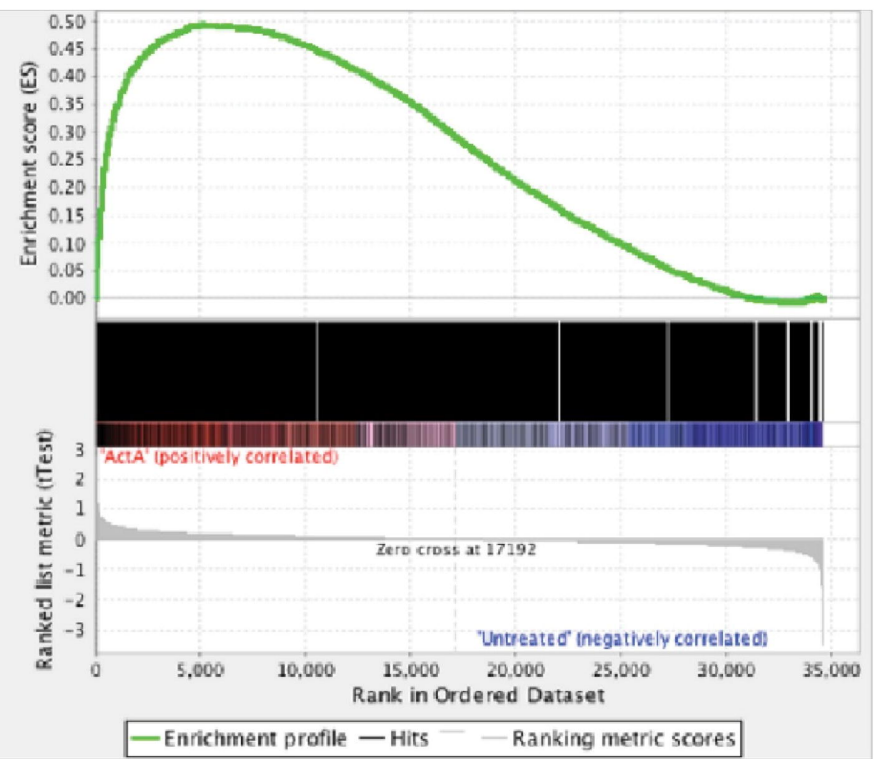

B

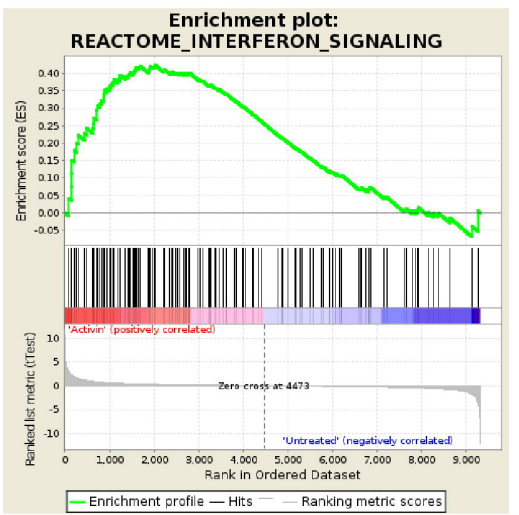

Enrichment plot: REACTOME_INTERFERON_GAMMA_SIGNALING
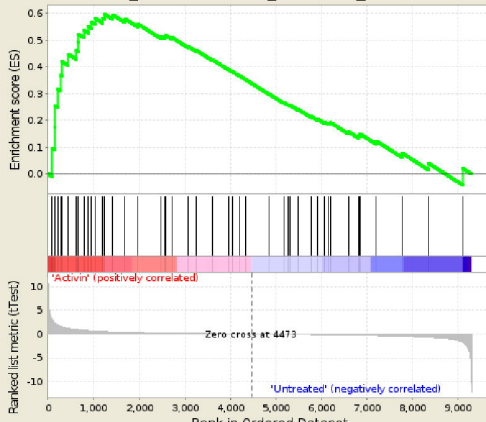
Rank in Ordered Dataset

- Enrichment profile — Hits - — Ranking metric scores 

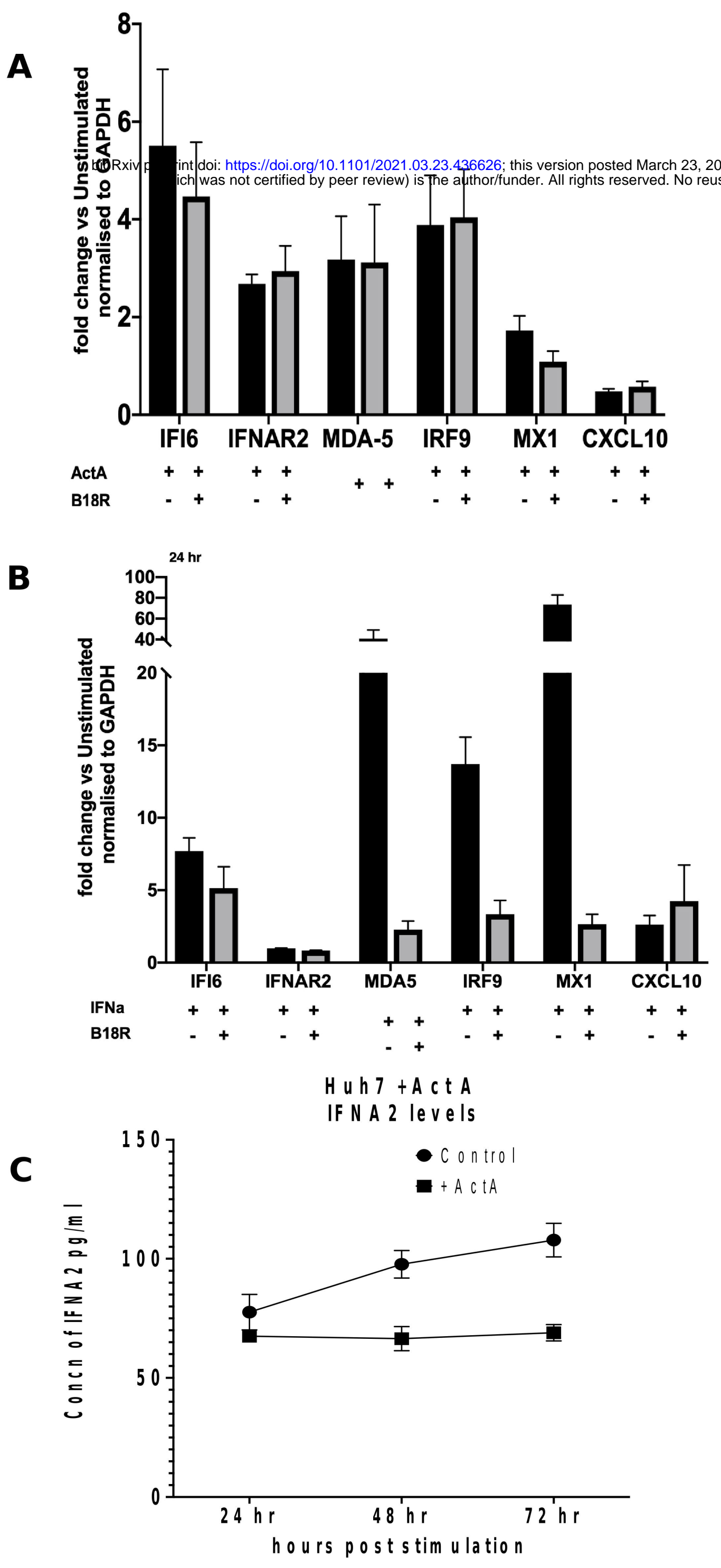
Control vs liver inflammation

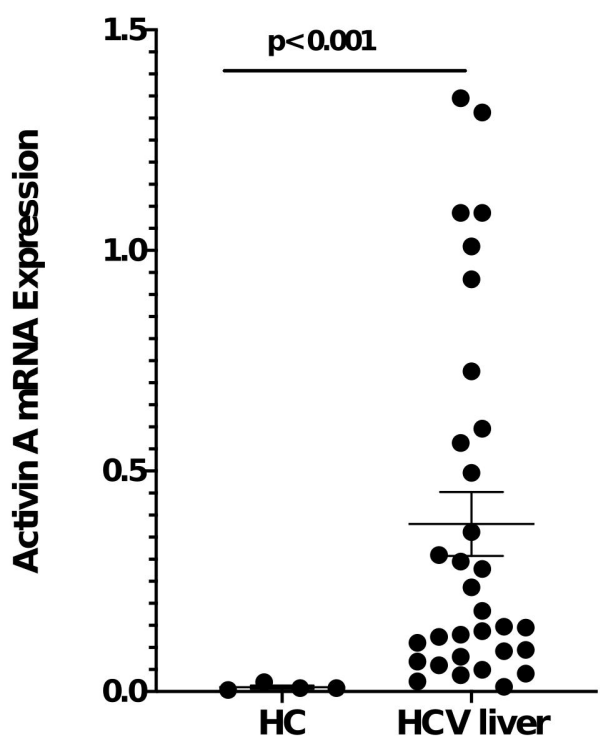

Act A ELISA in HCV patients

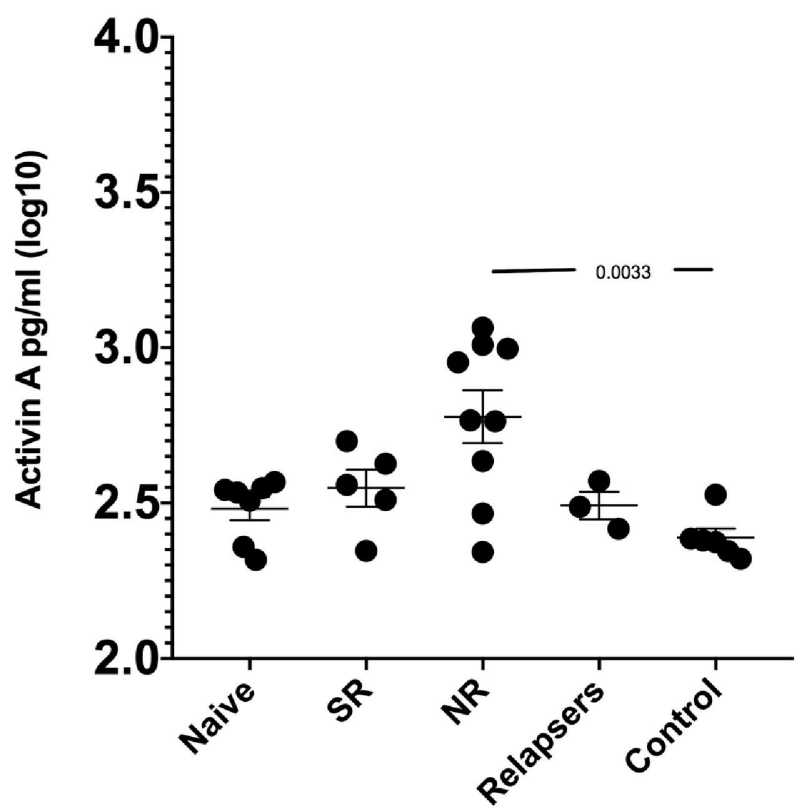



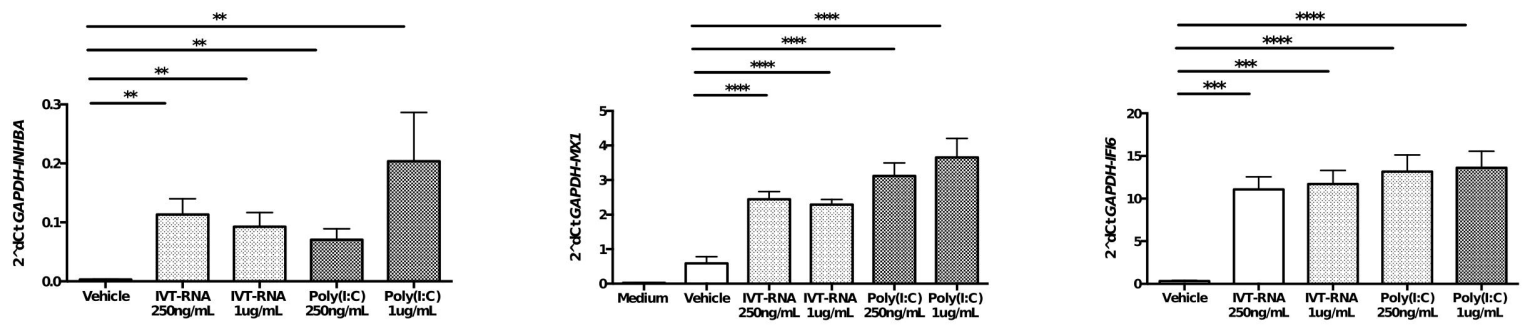

D

E

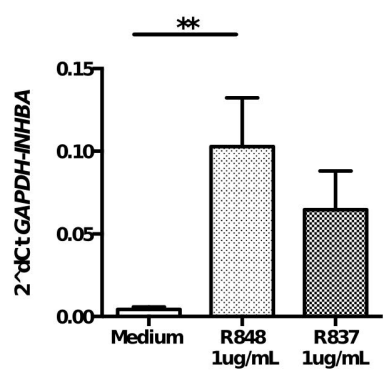

$\mathrm{H}$

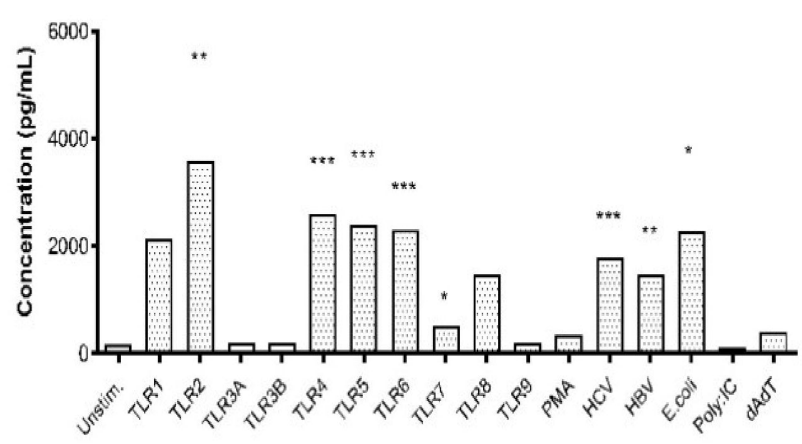

F

$G$
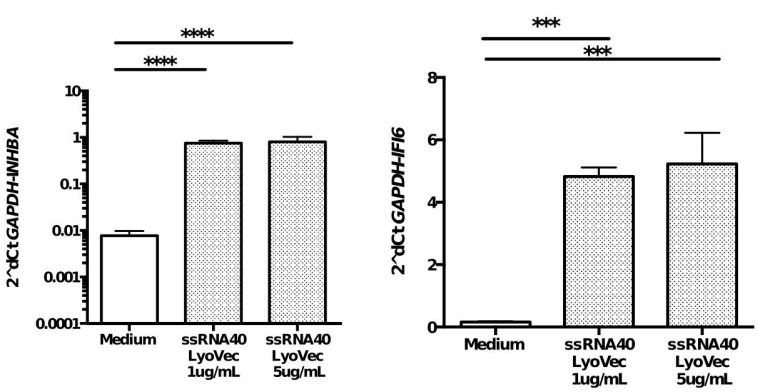

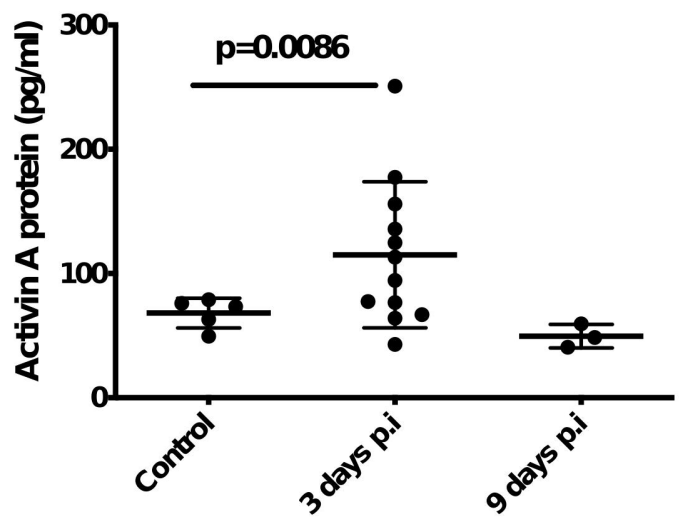




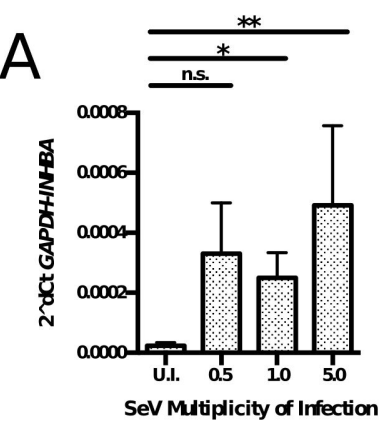

C
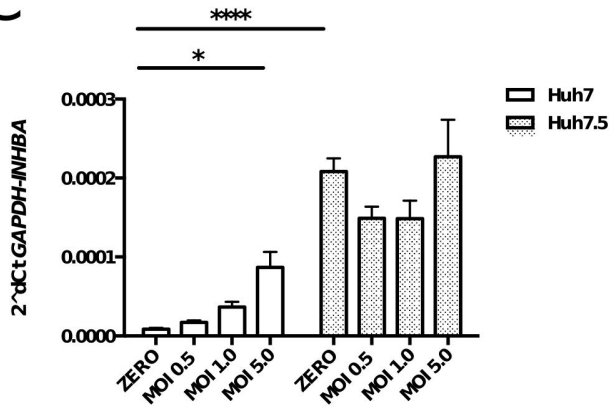

SeV Multiplicity of Infection
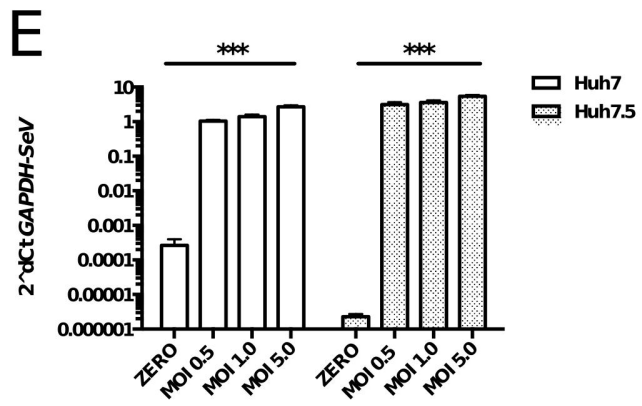

D

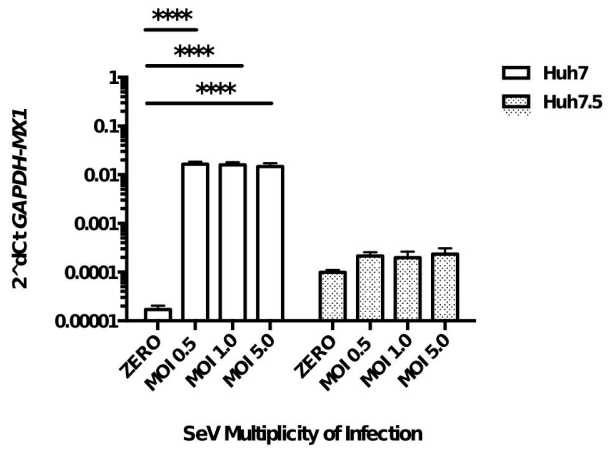

F

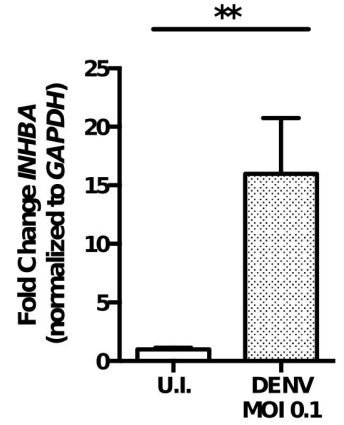

$G$

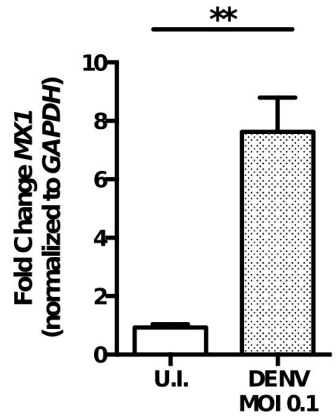




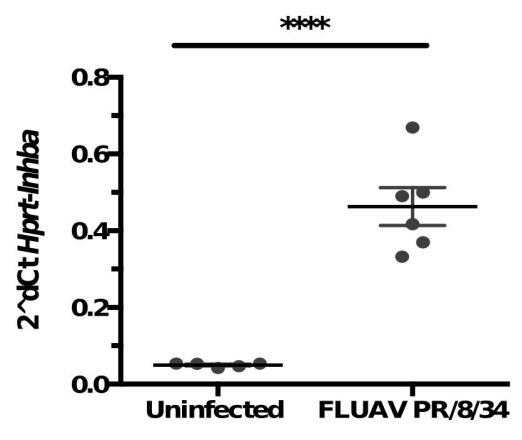

D

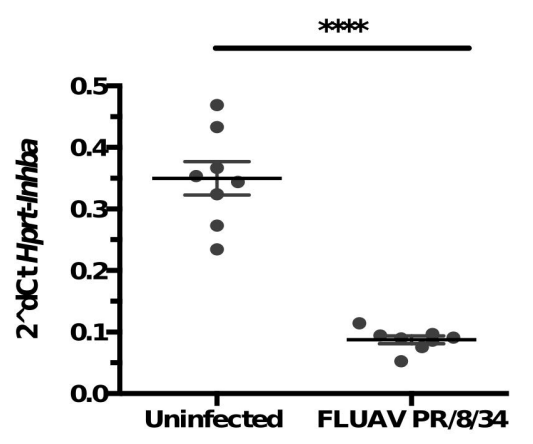

$\mathrm{F}$

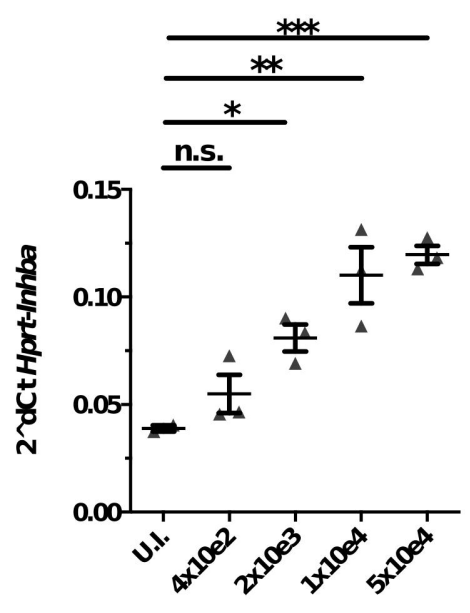

Influenza A PR/8/34 (pfu)

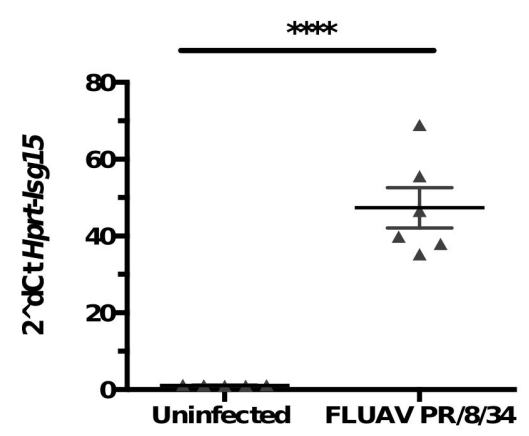

E

n.s.

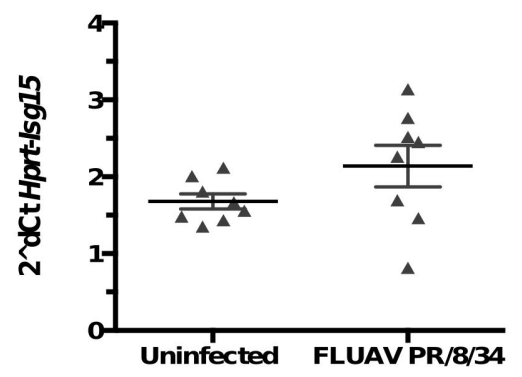

G

$\mathrm{H}$

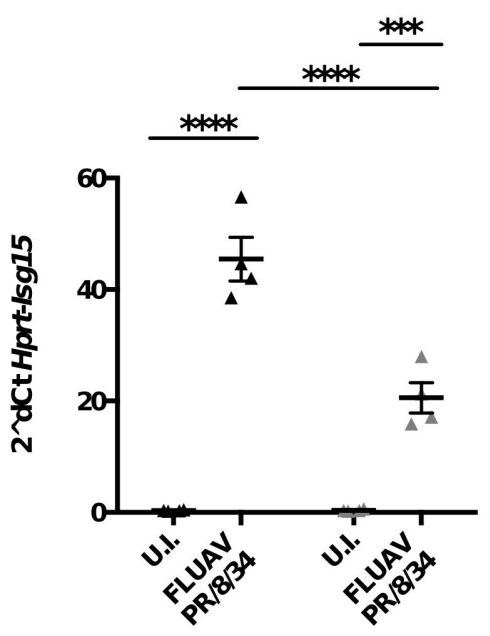

Wildtype

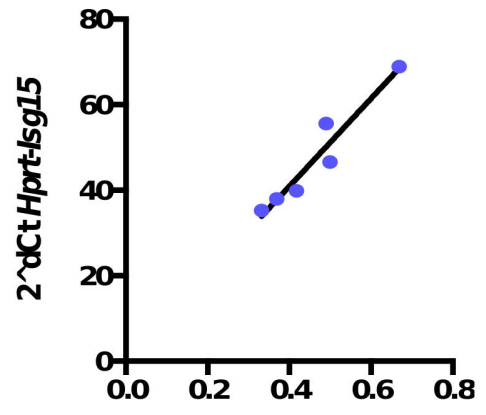

2 dCt Hprt-Inhba

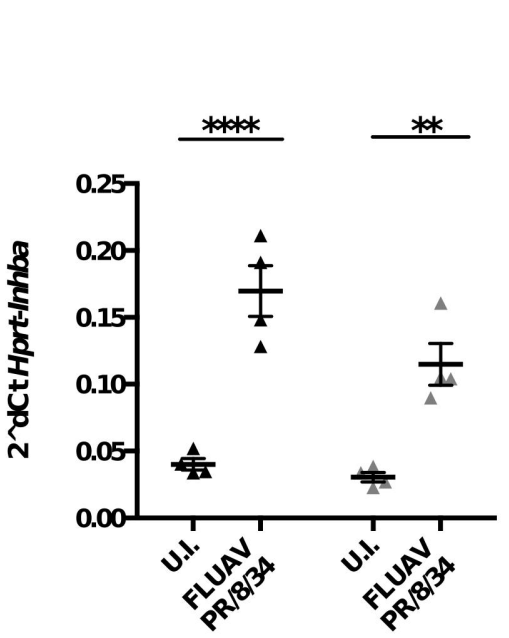

Wildtype 


\section{Figure 1:}

A) Diagram representing experimental cell culture procedure on stimulation of HepaRG cells with Activin A.

B)C) Metacore analaysis performed on differentially regulated genes identified by microrray analysis of RNA from HepaRG cells stimulated with 10nM activin A over 24 hours. Figure 1B highlights pathways mapped to the 184 signficantly upregulated genes; figure $1 \mathrm{C}$ highlights those mapped to the 168 significantly downregulated transcripts.

D) Time course over a period of 72 hours of HepaRG cells stimulated with Activin A (10nM), with relative gene expression levels of various ISGs shown compared to unstimulated cells. $n=3$ independent experiments conducted in triplicate.

E) Comparative analysis of gene expression in different hepatocyte cell lines (HuH7, HepaRG, HHL12) at 24 hours following stimulation with Activin A (10nM). $n=3$ independent experiment conducted in triplicate.

\section{Figure 2:}

A) Gene Set Enrichment Analysis (GSEA) showing consistency of results between microrray and RNA-sequencing experiments interrogating transcriptomes elicited by treatment of HepaRG hepatoma-derived cells with activin A 10nM over a period of 24 hours. The whole set of upregulated genes $(\log \mathrm{FC}>0, \mathrm{p}$ value $>0.1, \mathrm{n}=2571)$ between Activin vs Untreated in RNA-seq were found significantly enriched (FDR $=0.04, \mathrm{p}<<0.01$ ) in Activin-treated samples derived from the equivalent microarray experiment described in Figure 1A (Activin vs Untreated condition).

B)C) GSEA showing enrichment of Interferon gene pathways in transcriptomics from Activin treated samples. Based on 110 Interferon-related signatures selected from MsigDb, two representative Interferon Gene Sets were defined, corresponding to Interferon Signalling (Figure 2B) and Interferon Gamma Signalling. Both of these gene sets were founds to be significantly enriched in RNA-derived from HepaRG cells stimulated with activin A 10nM over 24 hours (Figure 2A: FDR $=0.19, \mathrm{p}=0.026$; Figure 2B: $\mathrm{FDR}=0.019, \mathrm{p}=0.004$ respectively.

\section{Figure 3:}

A)Gene Set Enrichment Analysis performed on two sets of genes obtained by RNA sequencing and microarray analysis. Results show high correlation in gene expression by both methods of analysis. Metacore analysis showing genes from significant canonical pathways in hepatocytes stimulated with Activin A, BMP6 and IFNa respectively.

Metacore analysis showing genes from significant process network in hepatocytes stimulated with Activin A, BMP6 and IFNa respectively

\section{Figure 4:}

A) Activin A mRNA expression in patients with liver inflammation due to $\mathrm{HCV}$ infection, each assay done in duplicate. Patients were graded based on their ISHAK scores $n=32$. P-values obtained via Mann-Whitney U-test.

B) Activin A protein in HCV serum samples as detected by ELISA. Naive (treatment naive) ( $\mathrm{n}=7$ ), SR (spontaneous resolvers)( $\mathrm{n}=5), \mathrm{NR}$ (non responders)(n=9). P-values obtained via unpaired t test.

\section{Figure 5:}


A) B) C) In human PBMC, INHBA is significantly induced by transfected poly(I:C) and poly(dA:dT) at two doses, in parallel with significant upregulation of the ISGs MXI and IFI6 across all transfection conditions. D) E) INHBA is significantly induced by R848 exposure in human PBMC, with non-significant upregulation elicited by R837. MX1 is significantly induced by both ligands. F) G) In human PBMC, both INHBA and MXI message are significantly elevated following transfection with the TLR8 agonist ssRNA40. For INHBA quantification, $\mathrm{n}=9$ donors; for $M X 1, \mathrm{n}=6$ donors; for $I F I 6, \mathrm{n}=3$ donors. Samples transfected in singlicate; mean+SEM presented; analysis by multiple unpaired, two-tailed t-tests. H) Activin A protein levels as determined by ELISA on supernatants of CD14+ cells from n=3 donors stimulated with TLR agonists for $24 \mathrm{hrs}$. I) Activin A levels in serum of mice infected with MCMV.

\section{Figure 6:}

A) B) Both INHBA and $M X 1$ expression are significantly induced in A549.gfp cells infected with increasing titres of $\mathrm{SeV}$ compared to uninfected controls, at 48 h.p.i. For both $I N H B A$ and $M X 1, \mathrm{n}=3$ independent experiments, each in biological duplicate; mean+SEM; analysis with ordinary one-way ANOVA and Bonferroni's post-test with respect to the uninfected condition. C) D) E) At $48 \mathrm{~h}$, in the Huh7 line, INHBA mRNA is dose-dependently induced by increasing $\mathrm{SeV}$ titre, whereas no induction occurs in RIG-I deficient Huh7.5 cells. In parallel, $M X 1$ is profoundly upregulated upon $\mathrm{SeV}$ infection of Huh7 cells, but is not elevated in the RIG-I deficient Huh7.5.1 line (B). SeV genomic RNA is detected at robust levels upon infection at all titres in both cell line. $n=2$ independent experiments, each in biological duplicate; mean + SEM; analysis by two-way ANOVA and Bonferroni's multiple comparisons test with respect to uninfected cells. F) G) Both INHBA and $M X 1$ are significantly upregulated in DENV-infected Huh7.5.1 cells relative to uninfected (U.I.), at 72 h.p.i. For $I N H B A, \mathrm{n}=4$; for $M X 1, \mathrm{n}=3$ independent experiments, each in biological triplicate; mean+SEM; analysis by unpaired two-tailed t-tests.

\section{Figure 7:}

A) B) C) Inhba and Isg $15 \mathrm{mRNA}$ are upregulated in the lungs of FLUAV PR/8 infected mice at 72 h.p.i. The degree of their respective induction is strongly positively correlated $\left(\mathrm{R}^{2}=0.9271\right.$, $\mathrm{p}=0.0020$ ). $\mathrm{n}=5$ uninfected and $\mathrm{n}=6$ infected mice; mean+SEM; analysis by unpaired two-tailed $\mathrm{t}-$ test (A,B) and linear regression (C). D) E) At 72 h.p.i. in the liver, Inhba message is significantly suppressed in the infected mice, whereas hepatic levels of Is 15 message do not differ between groups. $\mathrm{n}=8$ mice per group; mean+SEM; analysis by unpaired two-tailed t-test. F) Lung Inhba expression is dose-dependently induced by FLUAV infection at 48 h.p.i. $n=3$ mice per group; mean+SEM; analysis by ordinary one-way ANOVA and Bonferroni's post-test with respect to uninfected mice G) H) mRNA encoding both Inhba and Isg15 is significantly upregulated in the lungs of both WT and Mavs KO mice at 48 h.p.i. with FLUAV; no significant difference in the magnitude of induction is evident between groups.

\section{Supplementary Figure 1:}

A) $\mathrm{HuH} 7$ cells were incubated with recombinant IFN $\alpha$ 2a for $24 \mathrm{~h}$, followed by RNA extraction and RT-qPCR quantification of mRNA encoding INHBA. $\mathrm{n}=4$ independent experiments; mean + S.E.M.; analysis by two-tailed unpaired t-test.

B) C) D) Co-incubation with activin A synergistically enhances the antiviral effect of IFN $\alpha$ in the OR6 HCV replicon model. OR6 cells were incubated with a titration of recombinant human IFN $\alpha$ in the presence of titrating doses of recombinant human activin A; cells were assayed at $72 \mathrm{~h}$ postincubation for Renilla luciferase (A) and Cell Titer Glo (B), with signal presented relative to the untreated condition. Renilla luciferase signal is normalized to Cell Titer Glo intensity, to account for 
variation in cell number, in (C). Normalized for the dose-dependent diminution in cell number/viability caused by activin A, co-incubation with activin A synergistically enhances the antiviral effect of both $10 \mathrm{U} / \mathrm{mL}$ and $100 \mathrm{U} / \mathrm{mL}$ IFNa. $n=3$ independent experiments in biological triplicate; mean+SEM; analysis by 2-way ANOVA and Bonferroni's multiple comparisons test with respect to the ZERO activin A condition within each group. 